\title{
PRECAUTIONARY SAVINGS AS AN INSTRUMENT TO HEDGE THE RISK OF HYDROLOGICAL DROUGHT IN IRRIGATED AGRICULTURE
}

\author{
José A. Gómez-Limón*, M. Dolores Guerrero-Baena and José A. Fernández-Gallardo \\ WEARE-Water, Environmental and Agricultural Resources Economics Research Group, University of \\ Cordoba, (jglimon@uco.es, dolores.guerrero@uco.es, jose.fernandez@uco.es, Cordoba,Spain)
}

\begin{abstract}
This paper explores the use of precautionary savings as a new risk management instrument that can be implemented to hedge hydrological drought risk in irrigated agriculture, a risk currently not covered by any policy instrument. For this purpose, the Drought Savings Account (DSA) is proposed as a personal savings account to which farmers make regular contributions, with withdrawals allowed in the event of irrigation water supply gaps in order to guarantee a minimum income. The implementation of the DSA is empirically assessed in a Mediterranean-climate irrigation district using an innovative simulation approach. Based on the results obtained, the DSA has proved to be a theoretically suitable policy instrument that can overcome the problems hindering the implementation of agricultural insurance, managing the risk in a more costeffective way. This cost-effectiveness is a key advantage of precautionary savings over agricultural insurance, since the former instrument minimizes moral hazard and adverse selection problems, and the transaction costs of risk-sharing. Moreover, in this context, precautionary savings may play a significant role as an efficient climate change adaptation measure since the self-insurance strategy adopted does not distort the signals underlying farmers' own risk exposure, leading to better individual assessment and an adequate management of water supply gaps.
\end{abstract}

Keywords: Irrigation water supply gaps, Drought savings account, Hydro-economic stochastic modeling, Stochastic dominance analysis with respect to a function, Spain

\section{Introduction}

Agriculture is an economic activity that is characterized by its high exposure to different sources of risk that entail income and wealth losses to farmers. In Mediterranean-climate farming regions, such as in Spain, irrigation farmers are particularly concerned about droughts [Antón y Kimura (2011)]. When a hydrological drought episode occurs, instream flows and reservoir levels are not enough to meet all water needs, and irrigators are not allowed to use the full water allotments granted to them according to their water rights, suffering from 'water supply gaps', what lead to large losses [OECD (2016)]. Irrigators' awareness of the need to cope with these gaps is being reinforced by the increasing effect of climate change.

Currently, no risk management instrument specifically addresses the risk of hydrological drought in irrigated agriculture. Some policy-makers [OECD (2016); EC (2017)] and researchers [Colson et al. (2014); van Asseldonk et al. (2019)] have recently drawn attention to the use of precautionary savings as a potential and cost-effective risk management instrument in agriculture, aiming to overcome some of the problems linked to agricultural insurance. Some of the advantages of precautionary savings compared to agricultural insurance relate to its lower costs mainly due to there is no need to evaluate farm-level risk neither farm damages, while moral hazard and adverse selection problems are minimized.

The purpose of this paper is to explore the suitability of precautionary savings as an effective risk management instrument to cover the risk of hydrological drought in irrigated agriculture. For this purpose, two specific objectives are considered. First, the paper aims to present the design of a new, technically feasible personal precautionary savings account targeted at irrigation farmers voluntarily seeking to cope with the risk of hydrological drought: the Drought Savings Account (DSA). The proposed DSA is conceived as a risk management instrument aimed at guaranteeing a minimum level of farm income to farmers when a hydrological drought episode occurs. Second, the performance of the proposed DSA is simulated for an irrigation district in the Guadalquivir River Basin (southern Spain). The purpose of this case study is to analyse the effectiveness of precautionary savings as a hydrological drought risk management instrument in a real-life setting. This second objective contributes to the existing literature by proposing an innovative methodological approach combining hydro-economic stochastic modelling and farmers' utility gains derived from the change in the probability distribution function (PDF) of annual farm income (without and with the DSA) using the stochastic dominance analysis with respect to a function (SDRF). 


\section{The Drought Savings Account}

In Spain, water rights granted to irrigators fix the maximum annual volume (full water allotment, $f w a$ ) that these rights holders are allowed to extract from a specific water body. However, the volume of water actually available for these rights holders every year $t$ is uncertain, with the annual water allotment $\left(\widetilde{W A}_{t}\right)$ being set annually by the River Basin Authority (RBA) depending on the hydrological situation.

In 'normal' hydrological years expected farm income with full water allotment is $\bar{I}$. But, in water-scarce years (hydrological droughts) the RBA has to ration water allotments $\left(\widetilde{W A_{t}}<f w a\right)$. Thus, farm income in year $t\left(I_{t}\right)$ depends on irrigation water availability this year, both variables related through $f: I_{t}=f\left(\widetilde{W A_{t}}\right)$.

The DSA is designed to be voluntarily uptaken to guarantee that farmers get at least a predefined share of the expected farm income with full water allotment: $I^{g}=\alpha \cdot \bar{I}$, with $\alpha$ being a share of $\bar{I}$. This account operates with a single cash-flow a year (contribution or withdrawal at the end of the agricultural year), depending on the farm income obtained: i) when $\widetilde{W A}_{t} \geq c \cdot f w a$ ( $c$ denoting contribution), farmers are allowed to pay into the DSA with a regular annual contribution $\beta \cdot \bar{I}$; and ii) when $\widetilde{W A_{t}}<w \cdot f w a$ ( $w$ denoting withdrawal), farmers are allowed to withdraw funds to reach the farm income guaranteed (i.e. $I^{g}-I_{t}$ ).

The DSA could be also fed by public contributions $(\delta)$ matching farmers' contributions: $\delta \cdot \beta \cdot \bar{I}$. Moreover, DSA balances are affected by interest rates levied on deposits ( $r_{d}$, for positive balances) and money borrowed $\left(r_{b}\right.$, for negative balances). Considering usual banking practices, $r_{d}<r_{b}$. In order to avoid $D S A_{t}$ with large positive or negative balances, there should be an upper $(u)$ and lower limit $(-l)$ on $D S A_{t}$ to ensure rational risk coverage. It is also proposed that in the case of negative balances $\left(D S A_{t}<0\right)$, farmers must make additional contributions in the following years $(\gamma \cdot \bar{I})$, at least until their balance becomes positive, with the farmers' total annual contribution being $(\beta+\gamma) \cdot \bar{I}$.

Mathematically, the farmer's regular contribution to the DSA in year $t\left(F C_{t}\right)$ can be calculated using the following equation, where $C_{t}\{\cdot\}$ is an indicator function that equals 1 if the farmer must contribute to the DSA in year $t$, and 0 otherwise:

$$
F C_{t}=C_{t}\{\cdot\} \times \min \left\{\beta \cdot \bar{I}, \max \left[0,(1-\delta) \cdot\left(u \cdot \bar{I}-D S A_{t-1}\right)\right]\right\}
$$

When the farmer is requested to make an additional contribution to the account, that extra contribution $\left(E F C_{t}\right)$ can be modelled using the indicator function $E C_{t}\{\cdot\}$ (equaling 1 if the farmer must make an extra contribution, and 0 otherwise):

$$
E F C_{t}=E C_{t}\{\cdot\} \times \gamma \cdot \bar{I}
$$

The farmer's withdrawal from the DSA in year $t\left(F W_{t}\right)$ can be obtained using the following equation (denoting $W_{t}\{\cdot\}$ as an indicator function that equals 1 if the farmer can withdraw funds from the DSA in year $t$, and 0 otherwise):

$$
F W_{t}=W_{t}\{\cdot\} \times \min \left\{I^{g}-I_{t}, l \cdot \bar{I}+D S A_{t-1}\right\}
$$

Thus, the balance of the DSA in year $t$ can be calculated as follows:

$$
D S A_{t}=(1+\delta) \cdot\left(F C_{t}+E F C_{t}\right)-F W_{t}+(1+r) \cdot D S A_{t-1}
$$

To simulate the performance of the proposed DSA instrument, we modelled periods of 30 years, including all the equations 30 times (from $t=1$ to $t=30$ ), starting from an initial value of $D S A_{t=0}=0$. Using @ Risk 7.6 software, this 30-year model was simulated for 100,000 iterations implementing Latin Hypercube sampling techniques for time series of the stochastic variable $\widetilde{W A}_{t}$ from $t=1$ to $t=30$ drawn from the hydrological simulation model built by Gómez-Limón (2020). We obtained the PDFs for the whole set of variables describing the DSA performance with 100,000 simulated observations for every year $t$, from $\mathrm{t}=1$ to $t=30$. In this paper we focus on the results obtained for $t=5$ (short to medium term). The variables (measured in Euros per hectare) considered in the simulation model were: i) farmer's annual contribution to the DSA; ii) public matching contribution; iii) farmer's annual withdrawal; and iv) DSA annual cashflow and DSA balance.

The DSA is a risk management instrument because it enables a change in the PDF of annual farm income, from an initial distribution $\left(I_{t}\right)$ to a more stable income distribution $\left(I_{t}^{D S A}\right)$. The assessment of this instrument should also take into account the farmer's utility gains derived from this change in the PDF. For this purpose, we used the SDRF approach proposed by Hardaker et al. (2004), assuming that farmers' attitude to risk can be modelled relying on the expected utility theory, and that the expected utility of any risky prospect can be measured in terms of certainty equivalent. The risk premium (RP) can be used to quantify the preference of individual farmers for the risky alternative represented by $I_{t}^{D S A}$ over the risky alternative represented by $I_{t}$. 


\section{Case study. The Sector BXII Irrigation District}

Sector BXII (henceforth SBXII) is an irrigation district (ID) located close to the mouth of the Guadalquivir river (Southern Spain). This irrigated area covers a total of 14,643 hectares, operated by 569 farmers (average farm size of 25.7 hectares). Due to its Mediterranean climate, the main cultivated crops are cotton, corn, sugar beet, wheat and vegetables such as tomatoes, carrots and onions. Irrigators are organized into a water users' association that collectively holds water rights allowing an annual maximum water use of 6,000 cubic metres per hectare (full water allotment). Annual water allotments are provided by the RBA from its reservoir network. Due to the recurrent drought events, the amount of water annually available for this ID is usually lower than the full water allotment, resulting in water supply gaps that negatively affect farm income. Table 1 presents the values used for the set of parameters defining the model simulating the potential performance of the proposed DSA for a case study farm type.

Table 1. Parameters used in the DSA simulation model

\begin{tabular}{lccc}
\hline \multicolumn{1}{c}{ Parameter } & Value for simulations & Parameter & Value for simulations \\
\hline Full water allotment $(f w a)$ & $6,000 \mathrm{~m}^{3} / \mathrm{ha}$ & $\widetilde{W A} t_{t}$ threshold for contrib. $(c)$ & $80 \%$ of $f w a$ \\
Expected farm income with $f w a(\bar{I})$ & $3,021 € /$ ha & $\widetilde{W A} A_{t}$ threshold for withd. $(w)$ & $59.9 \%-46.8 \%$ of $f w a$ \\
Farm income guaranteed $I^{g}(\alpha)$ & $70 \%-80 \%$ of $\bar{I}$ & Upper cap on DSA balance $(u)$ & $200 \%$ of $\bar{I}$ \\
Regular annual contrib. to DSA $(\beta)$ & $2 \%-4 \%-6 \%$ of $\bar{I}$ & Lower cap on DSA balance $(l)$ & $100 \%$ of $\bar{I}$ \\
Catch-up payment if $D S A_{t-1}<0(\gamma)$ & $5 \%$ of $\bar{I}$ & Interest rate for deposits $\left(r_{d}\right)$ & $0.0 \%$ \\
Public matching contribution $(\delta)$ & $0 \%-2 \%-5 \%$ of & Interest rate for borrowings $\left(r_{b}\right)$ & $5.0 \%$ \\
\hline
\end{tabular}

Source: Authors' own elaboration.

\section{Results and discussion}

A summary of the results obtained ( 6 out of 18 scenarios analysed) for the performance assessment of the DSA instrument in the short to medium term ( $t=5$ years) is shown in Table 2 . In order to assess the DSA as a risk management instrument for coping with hydrological drought, we first focus on the variable measuring the percentage of years that the farmer's withdrawals are capped (i.e. those when drought risk is not properly hedged). In this regard, it can be seen that in all scenarios proposed the percentage of years that the farmer's withdrawals are capped is below $0.3 \%$, evidencing the suitability of DSA as a useful risk management instrument. It is worth noting that where the farm income guaranteed is defined as $80 \%$ of average income with full water allotment ( $\alpha=80 \%$, see scenarios 2,8 and 14 in Table 2), the percentage of years that the farmer's withdrawals are capped is higher than $0.2 \%$, while where income guaranteed is defined as $70 \%$ of average income with full water allotment ( $\alpha=70 \%$ considered in the other scenarios), this percentage is negligible (below $0.1 \%$ in every case). This indicates that the instrument of the precautionary savings proposed is suitable for hedging both shallow losses $(\alpha=80 \%)$ and deep losses of farm income $(\alpha=70 \%)$. However, if the farmer's and public sector's contributions to the DSA are intended to be reasonably low, it is advisable to design DSA to cover only deep losses, fixing $\alpha=70 \%$.

The second variable worth analysing is the farmer's regular annual contribution to the DSA. When comparing scenarios, in those where $\alpha=70 \%$ and $\delta=2 \%$ (scenarios 5, 11 and 17 in Table 2) it can be observed that the range of values considered for $\beta$ provides fairly similar performance in terms of hydrological drought risk hedging (percentages of years that the farmer's withdrawals are capped around $0.05 \%$ ). This result is possible because of the catch-up contributions to minimize negative balances. This buffering mechanism can be seen to follow the evolution of the variable percentage of years with catch-up payments, increasing from $14.2 \%$ in Scenario $5(\beta=6 \%)$ to 21.4\% in Scenario $17(\beta=2 \%)$. These results evidence the actuarial soundness of the DSA for a wide range of the farmer's regular annual contributions. In any case, for the case study analysed here, the most suitable values of $\beta$ are around $4 \%$. This value is high enough to avoid the percentage of years when hydrological drought risk cannot be hedged rises above $0.1 \%$, to prevent regular negative DSA balances and to avoid regular high positive DSA balance.

Simulation results regarding public matching contribution, suggest that the performance of the DSA instrument is quite similar in all the scenarios where $\alpha=70 \%$ and $\beta=4 \%$ (whatever $\delta$ is, i.e, $0 \%, 2 \%$ or $5 \%$ ) both in terms of risk hedging and the average DSA final balance.

Finally, regarding the farmer's utility gains estimated using the SDRF approach, the implementation of the DSA would involve a sharp change in the PDF of farm income and gains in monetary terms using risk premia (even without public support). Moreover, comparing the public matching contribution with the increase in the farmer's utility gains, both measured in euros per hectare and year, would suggest small overall economic efficiency gains (public contributions are lower than the increases in risk premia). These results suggest that public support for the proposed DSA plays a secondary role, with a marginal effect on the efficiency of the DSA as a risk management instrument and farmers' willingness to adopt it. 
Therefore, all the abovementioned comments justify the selection of Scenario $11(\alpha=70 \%, \beta=4 \%$ and $\delta=2 \%$ ) as the most suitable option for the implementation of the proposed DSA in a real-life setting in the short to medium term.

Table 2. DSA performance ( $\mathrm{t}=5$ years) for selected design scenarios.

\begin{tabular}{|c|c|c|c|c|c|c|c|}
\hline \multirow[b]{2}{*}{ Farm income guaranteed $(\alpha)$} & & \multicolumn{2}{|c|}{ Scenario 2 Scenario 5} & \multicolumn{2}{|c|}{ Scenario 8 Scenario 1} & \multicolumn{2}{|c|}{ Scenario 14 Scenario 17} \\
\hline & & $80 \%$ & $70 \%$ & $80 \%$ & $70 \%$ & $80 \%$ & $70 \%$ \\
\hline Regular contribution DSA $(\beta)$ & & $6 \%$ & $6 \%$ & $4 \%$ & $4 \%$ & $2 \%$ & $2 \%$ \\
\hline Public matching contribution $(\delta)$ & & $2 \%$ & $2 \%$ & $2 \%$ & $2 \%$ & $2 \%$ & $2 \%$ \\
\hline Farmer's contribution ( $€$ /ha·year) & Mean & 130.7 & 124.9 & 94.5 & 87.9 & 58.6 & 51.7 \\
\hline Public matching contrib. ( $€ /$ ha $\cdot$ year $)$ & Mean & 2.6 & 2.5 & 1.9 & 1.8 & 1.2 & 1.0 \\
\hline Years with contributions (\%) & & $64.3 \%$ & $64.2 \%$ & $64.2 \%$ & $64.0 \%$ & $64.2 \%$ & $64.1 \%$ \\
\hline Years with catch-up payments (\%) & & $23.6 \%$ & $14.2 \%$ & $28.0 \%$ & $17.4 \%$ & $32.9 \%$ & $21.4 \%$ \\
\hline Years with withdrawals (\%) & & $21.1 \%$ & $13.8 \%$ & $21.1 \%$ & $13.9 \%$ & $21.1 \%$ & $13.8 \%$ \\
\hline Years with withdrawals capped (\%) & & $0.22 \%$ & $0.05 \%$ & $0.26 \%$ & $0.05 \%$ & $0.29 \%$ & $0.06 \%$ \\
\hline DSA final balance $(€ /$ ha $)$ & Mean & 29.2 & 291.1 & -167.0 & 93.9 & -364.7 & -101.0 \\
\hline Original distribution $I_{t}(€ /$ ha $\cdot$ year $)$ & $\begin{array}{l}\text { Mean } \\
\text { CV }\end{array}$ & $\begin{array}{c}2,711 \\
91.7\end{array}$ & $\begin{array}{c}2,711 \\
91.3\end{array}$ & $\begin{array}{c}2,711 \\
91.1\end{array}$ & $\begin{array}{c}2,708 \\
92.5\end{array}$ & $\begin{array}{c}2,711 \\
90.9\end{array}$ & $\begin{array}{c}2,711 \\
91.5\end{array}$ \\
\hline New distribution $I_{t}^{D S A}(€ / \mathrm{ha})$ & $\begin{array}{l}\text { Mean } \\
\text { CV }\end{array}$ & $\begin{array}{c}2,700 \\
29.5\end{array}$ & $\begin{array}{c}2,706 \\
40.4\end{array}$ & $\begin{array}{c}2,696 \\
31.8\end{array}$ & $\begin{array}{c}2,703 \\
44.4\end{array}$ & $\begin{array}{c}2,693 \\
34.4\end{array}$ & $\begin{array}{c}2,702 \\
47.9\end{array}$ \\
\hline Risk premium $(€ /$ ha·year $)$ & $\begin{array}{l}r_{r}=0.5 \\
r_{r}=4.0\end{array}$ & $\begin{array}{c}37.1 \\
302.5\end{array}$ & $\begin{array}{c}33.3 \\
264.8\end{array}$ & $\begin{array}{c}31.0 \\
288.5\end{array}$ & $\begin{array}{c}33.2 \\
257.0\end{array}$ & $\begin{array}{c}24.7 \\
277.5\end{array}$ & $\begin{array}{c}26.3 \\
243.0\end{array}$ \\
\hline Risk premium (\%) & $\begin{array}{l}r_{r}=0.5 \\
r_{r}=4.0\end{array}$ & $\begin{array}{c}1.4 \% \\
11.5 \%\end{array}$ & $\begin{array}{c}1.2 \% \\
10.2 \%\end{array}$ & $\begin{array}{c}1.2 \% \\
11.1 \%\end{array}$ & $\begin{array}{c}1.2 \% \\
10.0 \%\end{array}$ & $\begin{array}{c}0.9 \% \\
10.7 \%\end{array}$ & $\begin{array}{l}1.0 \% \\
9.5 \%\end{array}$ \\
\hline
\end{tabular}

$\mathrm{CV}$ : Coefficient of variation (ratio of the standard deviation to the mean).

Source: Authors' own elaboration.

\section{Concluding remarks}

The results obtained in this study suggest the actuarial soundness of the proposed DSA since it can provide a reasonable hedge of hydrological drought risk (farm income guaranteed is assured in more than $99 \%$ of the years) for a relatively wide range of regular annual contribution rates, being the catch-up contributions mechanism a key feature of the DSA. Moreover, the assessment of the DSA instrument has also revealed that precautionary savings lead to significant utility gains for adopters, even without public support, what may explain the current informal use of this tool among farmers. In any case, moderate public support is suggested to make it clear that agricultural risk management is a top policy priority, similar to agricultural insurance, and also to promote wider adoption, especially among more vulnerable farms. It is worth pointing out that, besides the abovementioned private utility gains, public subsidies on the DSA would be justified based on the substantial public benefits that may arise from the widespread adoption of this instrument.

\section{References}

Antón, J. y Kimura, S. (2011). "Risk Management in Agriculture in Spain”. OECD Food, Agriculture and Fisheries Papers, 43: 1-62.

Colson, G., Ramirez, O.A. y Fu, S. (2014). "Crop insurance savings accounts: a viable alternative to crop insurance?”. Applied Economic Perspectives and Policy, 36(3): 527-545.

EC (European Commission). (2017). Communication from the Commission to the European Parliament, the Council, the European Economic and Social Committee and the Committee of the Regions. The Future of Food and Farming. European Commission, Brussels.

Gómez-Limón, J.A. (2020). "Hydrological drought insurance for irrigated agriculture in southern Spain". Agricultural Water Management, 240: 106271.

Hardaker, J.B., Richardson, J.W., Lien, G. y Schumann, K.D. (2004). "Stochastic efficiency analysis with risk aversion bounds: a simplified approach". Australian Journal of Agricultural and Resource Economics, 48(2): 253-270.

OECD (Organisation for Economic Co-operation and Development). (2016). Mitigating Droughts and Floods in Agriculture. Policy Lessons and Approaches. OECD Publishing, Paris.

van Asseldonk, M., Jongeneel, R., van Kooten, G.C. y Cordier, J. (2019). “Agricultural risk management in the European Union: a proposal to facilitate precautionary savings". EuroChoices, 18(2): 40-46. 